\title{
Integrated optical readout for miniaturization of cantilever-based sensor system
}

Nordström, Maria; Zauner, Dan; Calleja, Montserrat; Hübner, Jörg; Boisen, Anja

Published in:

Applied Physics Letters

Link to article, DOI:

$10.1063 / 1.2779851$

Publication date:

2007

Document Version

Publisher's PDF, also known as Version of record

Link back to DTU Orbit

Citation (APA):

Nordström, M., Zauner, D., Calleja, M., Hübner, J., \& Boisen, A. (2007). Integrated optical readout for miniaturization of cantilever-based sensor system. Applied Physics Letters, 91(10), 103512.

https://doi.org/10.1063/1.2779851

\section{General rights}

Copyright and moral rights for the publications made accessible in the public portal are retained by the authors and/or other copyright owners and it is a condition of accessing publications that users recognise and abide by the legal requirements associated with these rights.

- Users may download and print one copy of any publication from the public portal for the purpose of private study or research.

- You may not further distribute the material or use it for any profit-making activity or commercial gain

- You may freely distribute the URL identifying the publication in the public portal

If you believe that this document breaches copyright please contact us providing details, and we will remove access to the work immediately and investigate your claim 


\title{
Integrated optical readout for miniaturization of cantilever-based sensor system
}

\author{
Maria Nordström ${ }^{\mathrm{a})}$ and Dan A. Zauner \\ MIC-Department of Micro and Nanotechnology, Nano DTU, Technical University of Denmark, Bldg. 345 \\ East, DK-2800 Kongens Lyngby, Denmark \\ Montserrat Calleja \\ Bionanomechanics Lab, Instituto de Microelectrónica de Madrid, C/Isaac Newton 8-PTM, Tres Cantos, \\ E-28760 Madrid, Spain \\ Jörg Hübner and Anja Boisen \\ MIC-Department of Micro and Nanotechnology, Nano DTU, Technical University of Denmark, Bldg. 345 \\ East, DK-2800 Kongens Lyngby, Denmark
}

(Received 22 May 2007; accepted 15 August 2007; published online 6 September 2007)

\begin{abstract}
The authors present the fabrication and characterization of an integrated optical readout scheme based on single-mode waveguides for cantilever-based sensors. The cantilever bending is read out by monitoring changes in the optical intensity of light transmitted through the cantilever that also acts as a waveguide. The complete system is fabricated in the photosensitive polymer SU-8. They show theoretical calculations on the expected sensitivity both when operated in air and liquid and compare these with experimental characterization of the system in air where the cantilever is deflected mechanically. The experimental results compare well with the results obtained from the theoretical calculations. (C) 2007 American Institute of Physics. [DOI: 10.1063/1.2779851]
\end{abstract}

Micrometer sized cantilevers were first developed with the invention of the scanning force microscope ${ }^{1}$ where the high sensitivity of these micrometer sized devices to interactions with nearby surfaces is taken advantage of. One branch of the cantilever research field has since then, via the advancements in atomic force microscopy targeted different sensing applications. ${ }^{2}$ The principle of detection is to monitor the bending of the cantilever due to the generated surface stress when the target is immobilized on one surface of the cantilever. Cantilever sensors find relevant applications such as detection of dichlordiphenyltrichlorethan (DDT) ${ }^{3}$ for environmental control and detection of explosives. ${ }^{4}$ For such applications it is beneficial to have a portable sensor system. To achieve true miniaturization the cantilevers must be fabricated with an integrated readout to avoid the use of bulky external measurement setups. One such approach has been to fabricate a piezoresistive readout scheme, which has been applied for both $\mathrm{Si}$ and polymer cantilevers. ${ }^{5,6}$ Here, we show the development of an integrated optical readout scheme where the cantilever deflection is read out by monitoring the intensity of light traveling through the system via the cantilever. A similar system has been presented by $\mathrm{Zi}$ noviev et al. ${ }^{7}$ with the cantilever fabricated in $\mathrm{SiO}_{2}$ and the waveguides structured in $\mathrm{Si}_{3} \mathrm{~N}_{4}$. However, $\mathrm{Si}$ cantilevers are very stiff which is a disadvantage when performing static deflection measurements and a cantilever material of a lower Young's modulus is desirable. SU-8 provides optimal mechanical properties while providing the means for fast fabrication by photolithography. Here we show a system with integrated optical readout completely fabricated in the polymer SU-8. SU-8 is also suitable for the optical features of the system because the coupling loss is only $0.25 \mathrm{~dB} /$ facet and the propagation loss is only $1 \mathrm{~dB} / \mathrm{cm}$ at $1310 \mathrm{~nm}$.
The wavelength of operation of this system is $1310 \mathrm{~nm}$ and the light is butt coupled into and out of the system via single-mode fibers with a core diameter of $9 \mu \mathrm{m}$ (Corning, USA). After entering the system, the light is guided via the $10-\mu \mathrm{m}$-wide input waveguide toward a region where the free-hanging cantilever is situated (Fig. 1). The input waveguide and the cantilever are in the same plane and when the light exits the input waveguide it travels across a 5- $\mu \mathrm{m}$-wide gap toward the cantilever. The light is coupled into the cantilever and continues, via the $10-\mu \mathrm{m}$-wide output waveguide, out of the system. As the cantilever bends, the amount of light that can couple into the cantilever waveguide is reduced and the intensity of the output light falls off. This work presents both a theoretical treatment of the sensitivity of the readout system as well as a comparison with experimental results.

The readout sensitivity of the sensor system is calculated by modeling the mode profiles of the four different regions the light passes through and applying overlap integrals in between them, using the Gaussian approximation of the mode profiles. ${ }^{8}$ The four regions are shown schematically with their respective dimensions and refractive indices in

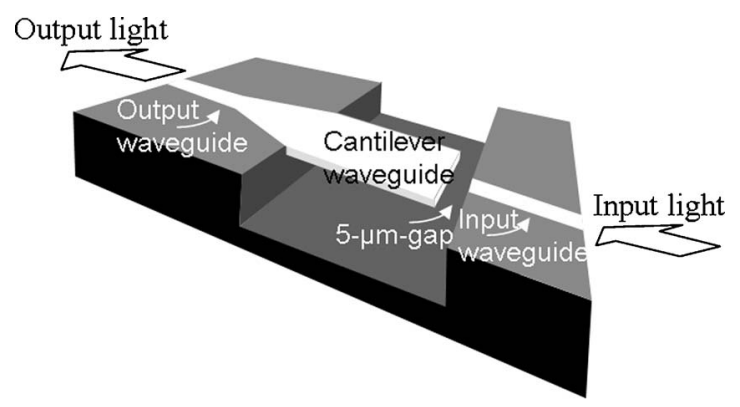

FIG. 1. Light travels through the system via the cantilever that also acts as a waveguide and it is collected in the opposite side.

\footnotetext{
${ }^{\text {a)} E l e c t r o n i c ~ m a i l: ~ m a n @ m i c . d t u . d k ~}$
} 


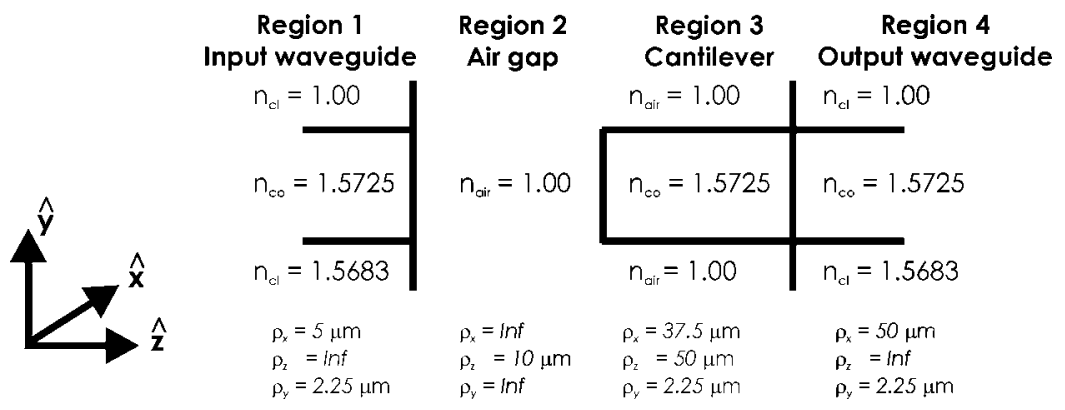

FIG. 2. Different regions with their respective refractive indices that the light travels through.
Fig. 2. The light mode is confined to a specific mode field diameter in regions 1,3 , and 4 due to the constant index step.

In region 2 the beam waist $w(l)$ can spread as it travels a distance $l$ from the input waveguide toward the cantilever, according to

$$
w(l)=w_{0}\left[1+\left(\frac{\lambda l}{\pi w_{0}^{2}}\right)^{2}\right]^{1 / 2},
$$

where $w_{0}$ is the initial beam waist and $\lambda$ is the wavelength of operation.

The coupling efficiency $\alpha$ is found from the expression

$$
\begin{gathered}
\alpha=\frac{\int_{-\infty}^{\infty} \int_{-\infty}^{\infty} \psi_{2}(x, y, l) \psi_{3}(x, y+d) d x d y}{\int_{-\infty}^{\infty} \int_{-\infty}^{\infty} \psi_{1}^{2}(x, y) d x d y} \\
\times \frac{\int_{-\rho_{x} \mathrm{wg}_{\text {out }}}^{\rho_{x} \mathrm{wg}_{\text {out }}} \int_{-\rho_{y}}^{\rho_{y}} \psi_{3}(x, y) \psi_{4}(x, y) d x d y}{\int_{-\rho_{x} \mathrm{cant}}^{\rho_{x} \mathrm{cant}} \int_{-\rho_{y}}^{\rho_{y}} \psi_{3}^{2}(x, y) d x d y},
\end{gathered}
$$

where $\psi_{1-4}$ is the mode profile in the respective regions, $l$ is the distance traveled, $d$ is the cantilever deflection, $\rho_{x} \mathrm{wg}_{\text {out }}$ is the half-width of the output waveguide, $\rho_{y}$ is the half-height of the cantilever-waveguide layer, and $\rho_{x}$ cant is the halfwidth of the cantilever.

At zero deflection of the cantilever and with air being the medium in between the cantilever waveguide and the output waveguide, approximately $40 \%$ of the input light is transmit-

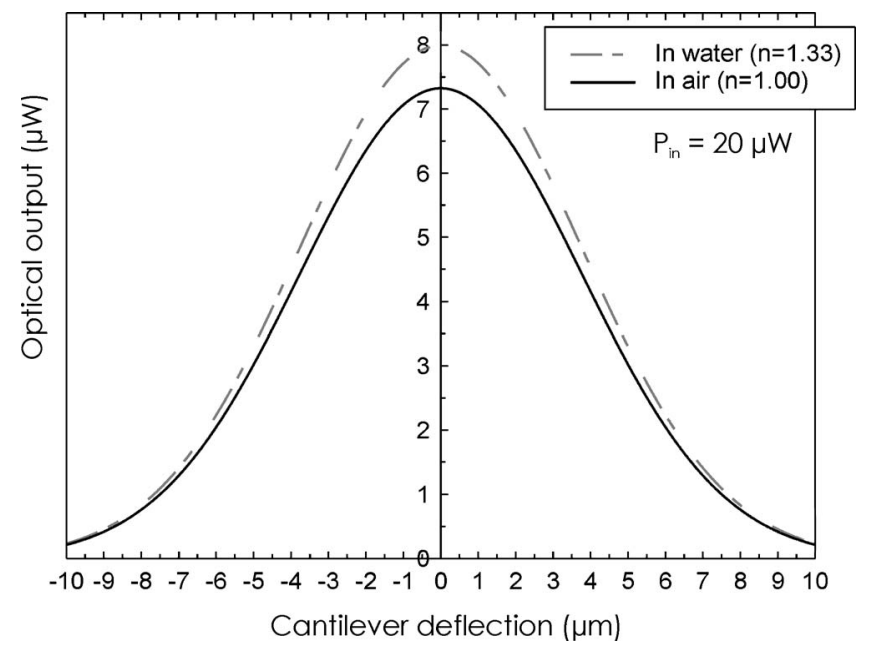

FIG. 3. When the medium between the cantilever waveguide and the output waveguide is water (or buffer solution) instead of air, the optical output is increased due to the reduced refractive index difference. increased due to the reduced refractive index difference. $\quad$ channel. ted through the system. Due to the large index step between the SU-8 and the air, the light is well confined inside the cantilever waveguide. Therefore it is assumed no significant radiation losses occur when the light travels through the cantilever, even at a maximum deflection of $9 \mu \mathrm{m}$. Figure 3 shows a comparison between the readout sensitivity when the intermediate medium is air or water. It can be seen that the coupling efficiency is increased when the medium is changed from air to water due to the reduced index contrast when the light couples into the cantilever waveguide.

The system is an all-polymer device. The cantilever and waveguide core are structured in SU-8 2005 (MicroChem, USA), which has been reported in the literature to have a low propagation loss combined with providing high cantilever sensitivity. $^{9-11}$ For cladding material the modified SU-8, mr-L 6050 XP (MicroResist, Germany) is used. This material combination has been shown to be highly suitable for the fabrication of micro-optoelectromechanical-system devices. ${ }^{12}$ Moreover, methods have been developed to immobilize biomolecules directly onto SU-8 without the need for a $\mathrm{Au}$ layer that usually results in large drifts due to the bimorph effect. ${ }^{11,13}$ Figure 4 shows a scanning electron microscope (SEM) image of the final chip with two cantilevers situated in the microfluidic channel.

The readout method is characterized by mechanically deflecting the cantilever a known distance while monitoring the optical output intensity. A $1310 \mathrm{~nm}$ laser source (HP 81552SM, Hewlett Packard, USA) is used as input and the intensity is detected by a lightwave multimeter (HP 81532A, Hewlett Packard, USA). The cantilever is deflected by pressing onto the apex with a tungsten probe mounted in a custom-made setup where the vertical displacement is controlled with submicron resolution. There is a drift in the system of $-0.2 \mathrm{nW} / \mathrm{s}$ due to a small drift of the micrometer screw and the photodetector has a noise level of $\pm 0.5 \mathrm{nW}$ when operated at this intensity level. Both these values are found experimentally from the measurements. Figure 5 shows a comparison between the theoretically calculated

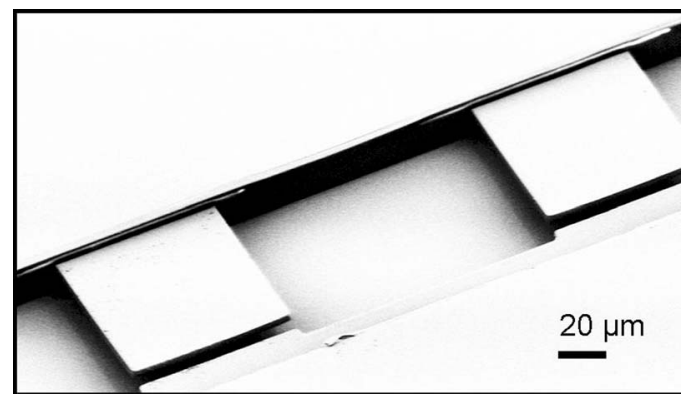

FIG. 4. SEM image of two free-hanging cantilevers in the microfluidic 


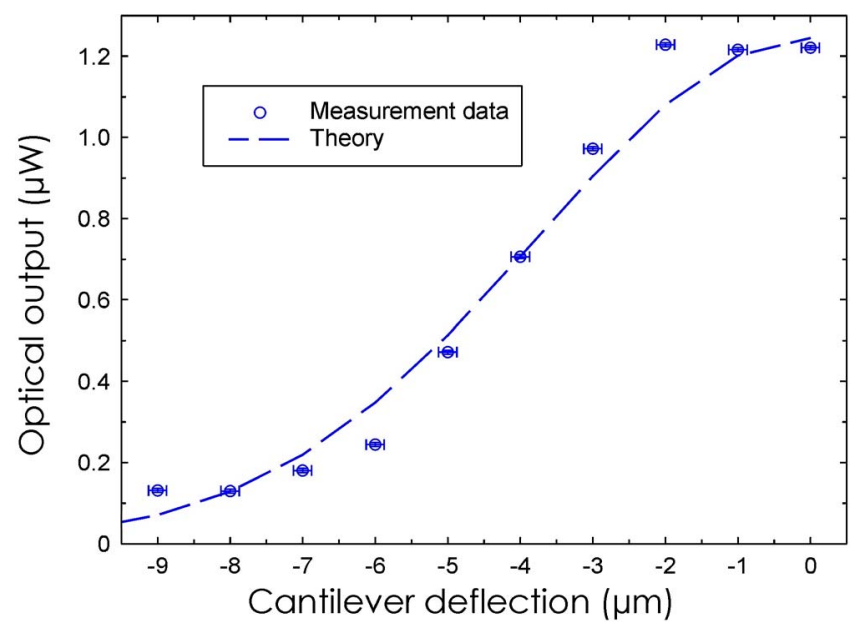

FIG. 5. (Color online) Comparison between calculated and measured values of the sensitivity. When operated in the most sensitive region, a cantilever deflection of $45 \mathrm{~nm}$ can be resolved.

readout and the experimentally obtained data points when operating the system in air.

In the most sensitive region, at a cantilever deflection of 3-5 $\mu \mathrm{m}$, the calculated minimum detectable cantilever deflection is found to be $45 \mathrm{~nm}$. This value is found by considering a noise level of $\pm 5 \mathrm{nW}$. The cantilever used in this experiment is $75 \mu \mathrm{m}$ wide, $100 \mu \mathrm{m}$ long, and $4.5 \mu \mathrm{m}$ thick, so this deflection corresponds to a surface stress of $0.19 \mathrm{~N} / \mathrm{m}$. This sensitivity can be further improved by using an optical setup with a lower noise level and by fabricating a longer cantilever since the length of the cantilever does not affect the coupling efficiency, whereas the cantilever deflection increases with the square of the length. ${ }^{14}$

This work shows the development of an integrated readout for cantilever-based sensors. The readout is based on fabricating a cantilever waveguide in the polymer SU-8. The use of a polymer enables increased cantilever sensitivity due to the low Young's modulus of the material. SU-8 is also well suited as waveguide material with a propagation loss of only $1 \mathrm{~dB} / \mathrm{cm}$ at $1310 \mathrm{~nm} .^{12}$ Moreover, the use of a polymeric material enables the direct binding of the analytes onto the cantilever. ${ }^{13}$ This readout mode offers a very straightfor- ward operation since it is only the intensity changes of the output light that is monitored without considering any phase changes or effects of birefringence. However, two aspects that have not been investigated yet and that might present an issue are the changes of the refractive index of the waveguide core upon surfaces stress changes of the cantilever and the continuous change of the mode profile of the cantilever waveguide with changes in the buffer solutions during operation.

Here, proof of principle is shown for the readout method by monitoring the output signal as the cantilever is mechanically deflected. A good fit is seen between theoretically calculated values and the data obtained experimentally from the characterization. The presented all-polymer system presents a great potential for the development of portable sensors with fully integrated and highly sensitive optical readout.

${ }^{1}$ T. R. Albrecht and C. F. Quate, J. Appl. Phys. 62, 2599 (1987).

${ }^{2}$ L. G. Carroscosa, M. Moreno, M. Álvarez, and L. M. Lechuga, Trends Analyt. Chem. 25, 196 (2006).

${ }^{3}$ M. Álvarez, A. Calle, J. Tamayo, L. M. Lechuga, A. Abad, and A. Montoya, Biosens. Bioelectron. 18, 649 (2003).

${ }^{4}$ L. A. Pinnaduwage, A. C. Gehl, S. L. Allman, A. Johansson, and A. Boisen, Rev. Sci. Instrum. 78, 055101 (2007).

${ }^{5}$ A. Boisen, J. Thaysen, H. Jensenius, and O. Hansen, Ultramicroscopy 82, $11(2000)$.

${ }^{6}$ A. Johansson, G. Blagoi, and A. Boisen, Appl. Phys. Lett. 89, 173505 (2006).

${ }^{7}$ K. Zinoviev, C. Domínguez, J. A. Plaza, V. J. Cadarso Busto, and L. M. Lechuga, J. Lightwave Technol. 24, 2132 (2006).

${ }^{8}$ F. Ladouceur and J. D. Love, Silica-Based Buried Channel Waveguides and Devices (Chapman and Hall, London, UK, 1996), 4, 32.

${ }^{9}$ J. M. Ruano-López, M. Aguirregabiria, M. Tijero, M. T. Arroyo, J. Elizalde, J. Berganzo, I. Aramburu, F. J. Blanco, and K. Mayora, Sens. Actuators B 114, 542 (2006).

${ }^{10}$ B. Bêche, N. Pelletier, E. Gaviot, and J. Zyss, Opt. Commun. 230, 91 (2004).

${ }^{11}$ M. Calleja, J. Tamayo, M. Nordström, and A. Boisen, Appl. Phys. Lett. 88, 113901 (2006).

${ }^{12}$ M. Nordström, D. A. Zauner, A. Boisen, and J. Hübner, J. Lightwave Technol. 25, 1284 (2007).

${ }^{13}$ R. Marie, S. Schmidt, A. Johansson, L. Ejsing, M. Nordström, D. Haefliger, C. B. V. Christensen, A. Boisen, and M. Dufva, Biosens. Bioelectron. 21, 1327 (2006).

${ }^{14}$ G. G. Stoney, Proc. R. Soc. London, Ser. A 82, 172 (1909). 